Supporting Information for

\title{
Stability and Compressibility of Cation-doped High-Entropy Oxide $\mathrm{MgCoNiCuZnO}$
}

Jian Chen ${ }^{1,2}$, Weixin Liu ${ }^{2}$, Junxiu Liu², Xiaoliang Zhang ${ }^{2}$, Mingzhi Yuan ${ }^{2}$,

Yunlei Zhao ${ }^{2}$, Jiejuan Yan²,3 Mingqiang $\mathrm{Hou}^{2,3}$, Jinyuan $\mathrm{Yan}^{3}$, Martin Kunz ${ }^{3}$, Nobumichi Tamura ${ }^{3}$, Hengzhong Zhang ${ }^{2, *}$, Zhoulan Yin ${ }^{1, *}$

1.College of Chemistry and Chemical Engineering, Central South University, Changsha, Hunan 410083, China.

2. Center for High Pressure Science and Technology Advanced Research, Shanghai 201203, China.

3. Advanced Light Source, Lawrence Berkeley National Laboratory, Berkeley, CA 94720, United States 


\section{Derivation of interatomic potential functions of some simple oxides}

The parameters of the Buckingham potential function (equation 1 in the main text) for the atomic pairs $\mathrm{Mg}-\mathrm{O}, \mathrm{Cu}-\mathrm{O}, \mathrm{Mn}-\mathrm{O}$ and $\mathrm{Li}-\mathrm{O}$ were derived out from empirical fitting to available elastic constants ${ }^{1-4}$ of the simple oxides $\mathrm{MgO}, \mathrm{CuO}, \mathrm{MnO}$ and $\mathrm{Li}_{2} \mathrm{O}$ using the program GULP ${ }^{5}$. For $\mathrm{MgO}$, the low- and high-frequency dielectric constants ${ }^{6}$ were also used. In the fitting, all the cations in the oxides were modeled as point charges having their formal charges: $\mathrm{Li}:+1, \mathrm{Mg}, \mathrm{Cu}$ and $\mathrm{Mn}: 2+$, whereas $\mathrm{O}$ was modeled using the shell model (see the main text). The obtained parameters are listed in Table S1.

Table S1. Fitted Me-O Potential Function Parameters of Some Simple Oxides

\begin{tabular}{|l|l|l|l|l|l|l|}
\hline Oxide & $\begin{array}{l}A \\
(\mathrm{eV})\end{array}$ & $\begin{array}{l}\rho \\
(\AA)\end{array}$ & $\begin{array}{l}c \\
\left(\mathrm{eV} \AA^{6}\right)\end{array}$ & $\begin{array}{l}\mathrm{B}_{0}(\mathrm{GPa}) \\
\text { Expt./Lit. }\end{array}$ & $\begin{array}{l}\mathrm{B}_{0}(\mathrm{GPa}) \\
\text { Fitted }\end{array}$ & $\begin{array}{l}\text { Ref. for } \\
B_{0} \text { (Expt./Lit.) }\end{array}$ \\
\hline $\begin{array}{l}\mathrm{Li}_{2} \mathrm{O} \\
\left(\text { cubic) }{ }^{\mathrm{a}}\right.\end{array}$ & 198.94 & 0.35807 & 0 & 93.6 & 95.4 & 1 \\
\hline $\begin{array}{l}\mathrm{MnO} \\
(\mathrm{cubic})^{\mathrm{b}}\end{array}$ & 583.65 & 0.35969 & 0 & 155.1 & 156.5 & 2 \\
\hline $\begin{array}{l}\mathrm{CuO} \\
(\mathrm{mono}))^{\mathrm{c}}\end{array}$ & 370.00 & 0.38922 & 0 & 73.9 & 73.3 & 3 \\
\hline $\begin{array}{l}\mathrm{MgO} \\
(\mathrm{cubic})^{\mathrm{d}}\end{array}$ & 5406.15 & 0.31342 & 490.287 & 163.8 & 162.5 & 4 \\
\hline
\end{tabular}

Crystallography Open Database. ${ }^{7}$

2. Additional EDXS elemental mapping of samples at relatively high resolutions

2.1 EDXS elemental mapping in undoped HEOx (area \#1)

Electron Image 3

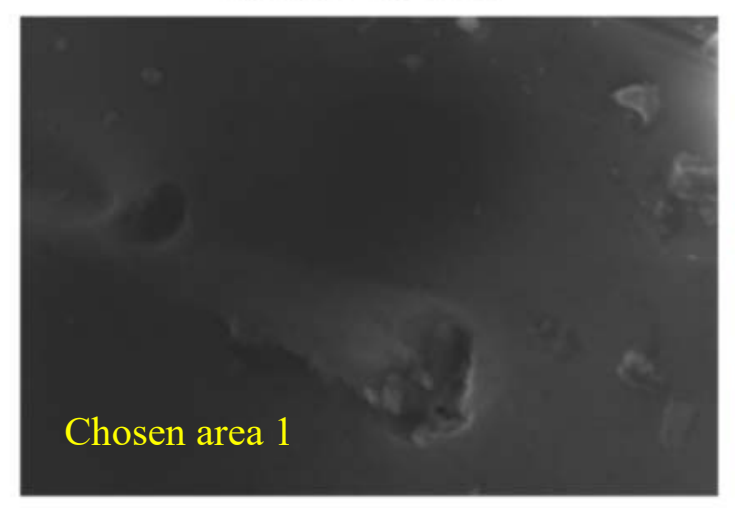

EDS Layered Image 1

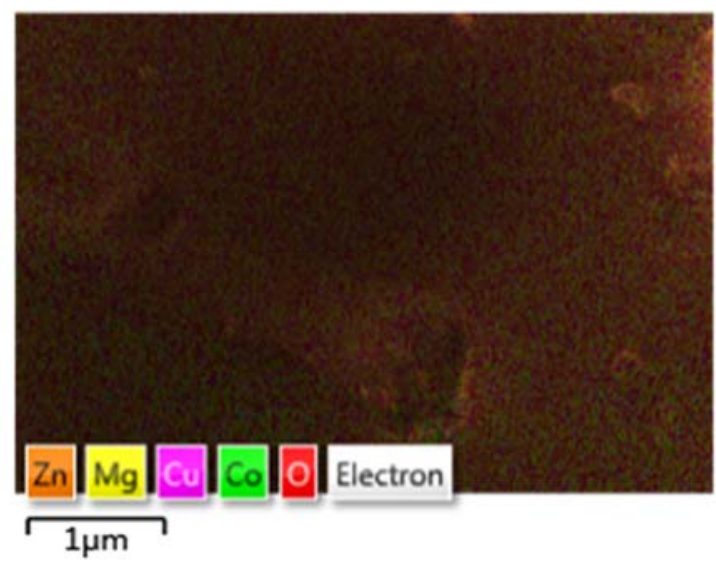


$\mathrm{Mg} \mathrm{K \alpha 1 \_ 2}$

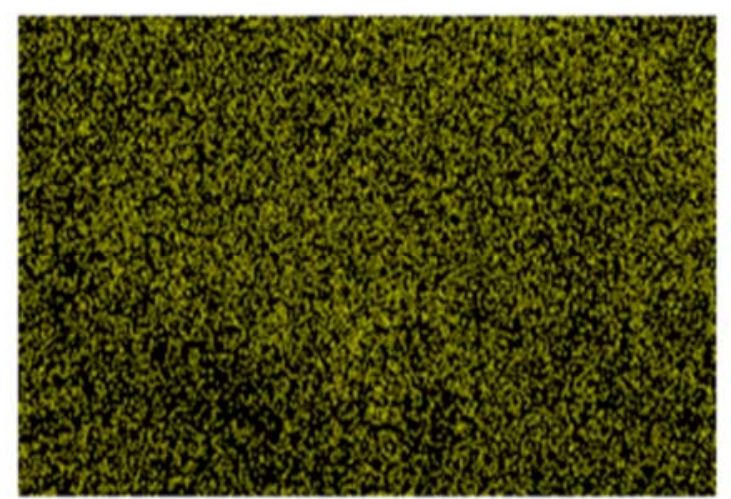

$\longdiv { 1 \mu \mathrm { m } }$

Ni K $\alpha 1$

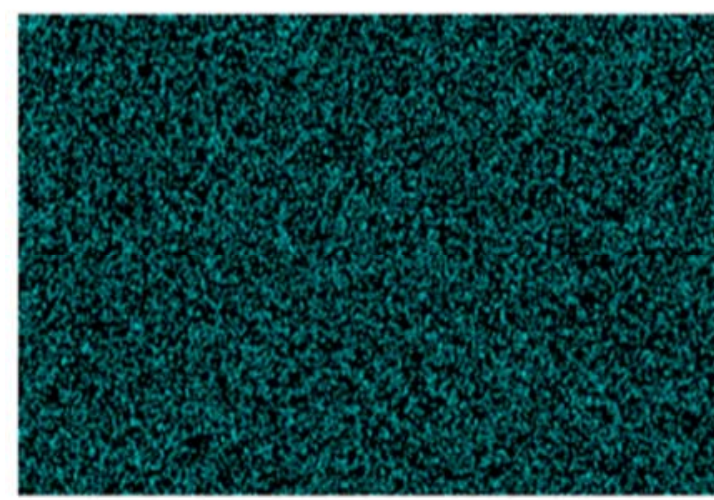

$1 \mu \mathrm{m}$

\section{Zn La1_2}

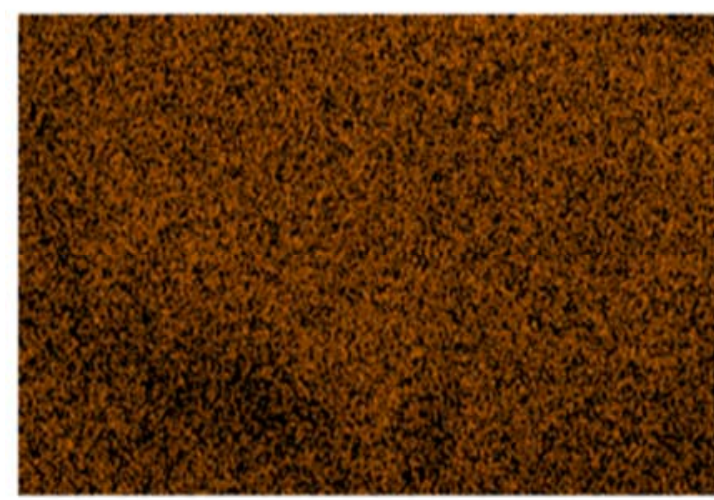

$1 \mu \mathrm{m}$
Co K $\alpha 1$

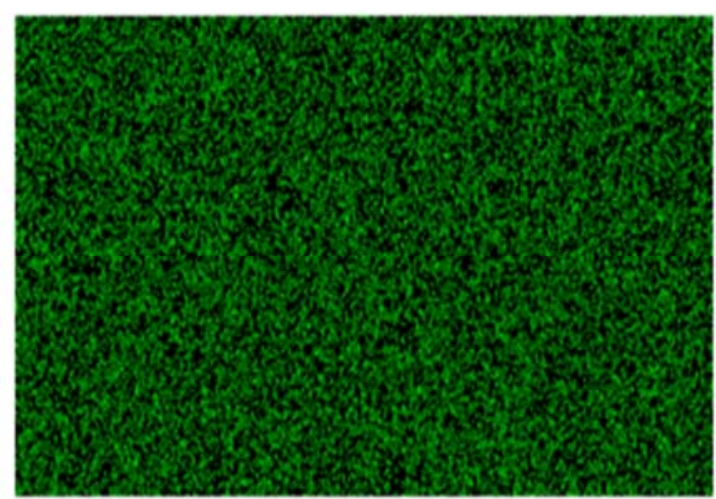

$\longdiv { 1 \mu \mathrm { m } }$

\section{Cu La1_2}

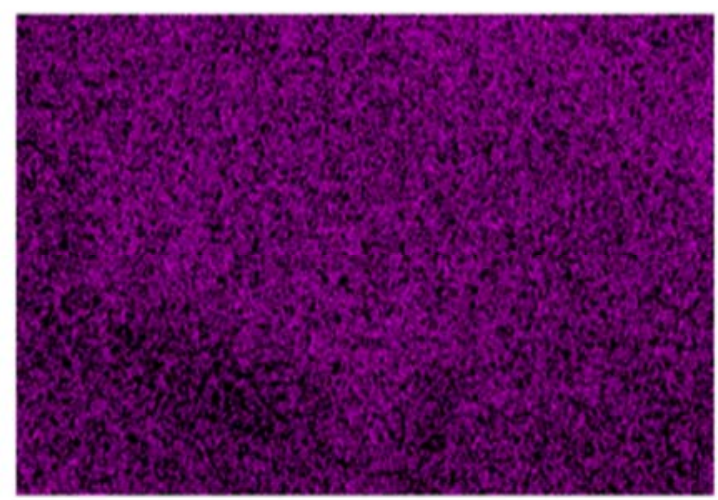

$\longdiv { 1 \mu \mathrm { m } }$

$\mathrm{O} K \alpha 1$

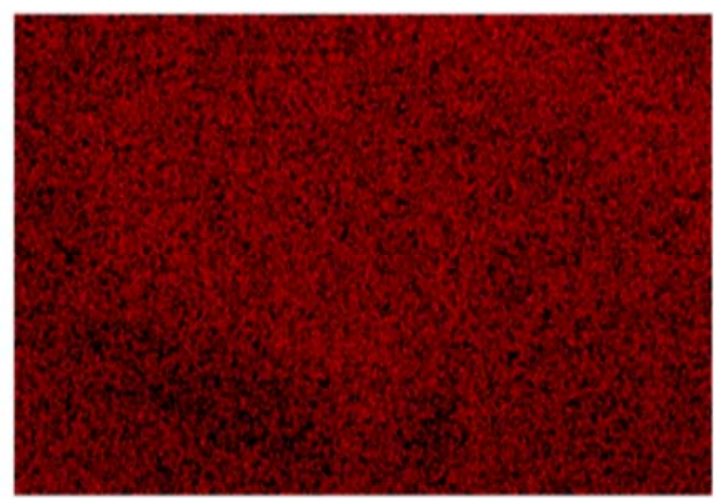

$\longdiv { 1 \mu \mathrm { m } }$

Figure S1. SEM image, EDXS layered image and EDXS elemental mapping in a chosen area $\# 1$ of undoped HEOx at a relatively high resolution (compared to Figure 1c). 
2.2 EDXS elemental mapping in undoped HEOx (area \#2)

Electron Image 4

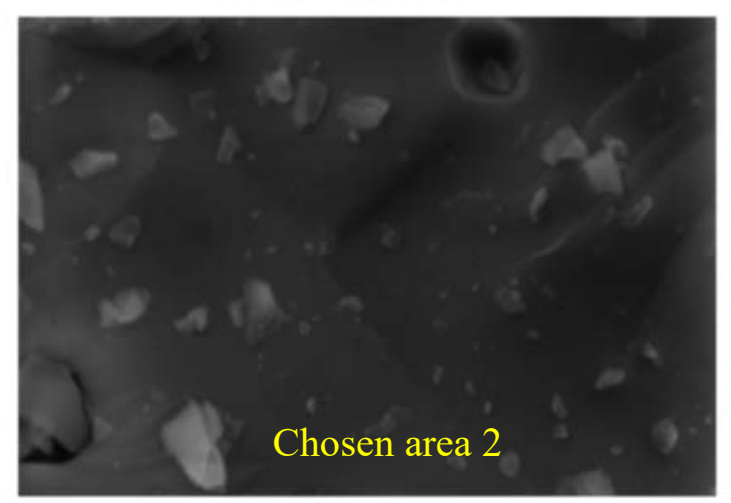

$\longdiv { 1 \mu \mathrm { m } }$

\section{$\mathrm{Mg} \mathrm{K} \alpha 1 \_2$}

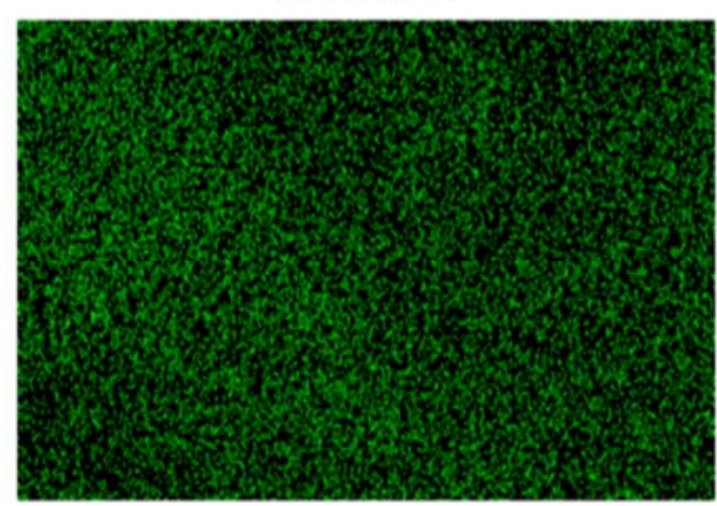

$\longdiv { 1 \mu \mathrm { m } }$

Ni K $\alpha 1$

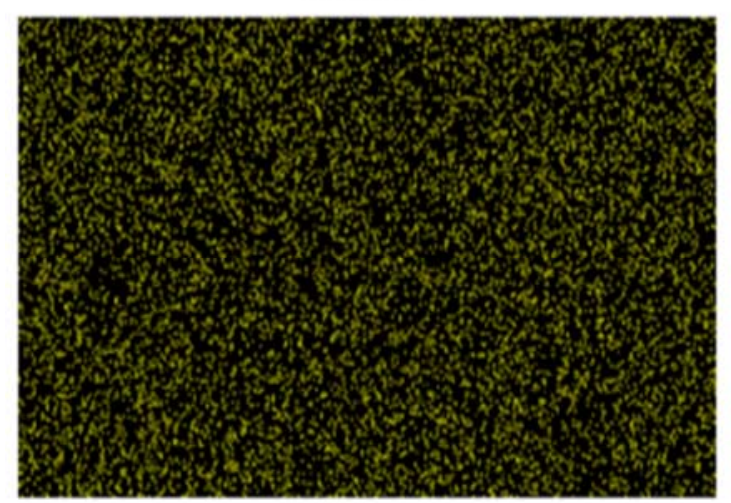

$\longdiv { 1 \mu \mathrm { m } }$
EDS Layered Image 1

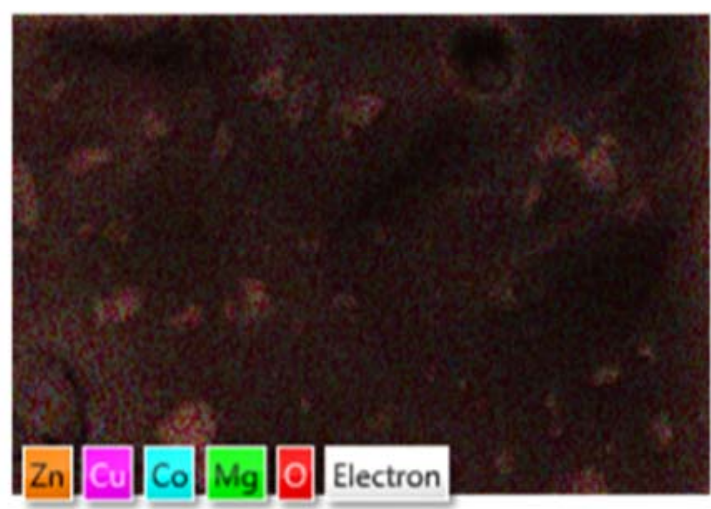

$\longdiv { 1 \mu \mathrm { m } }$

Co K $\alpha 1$

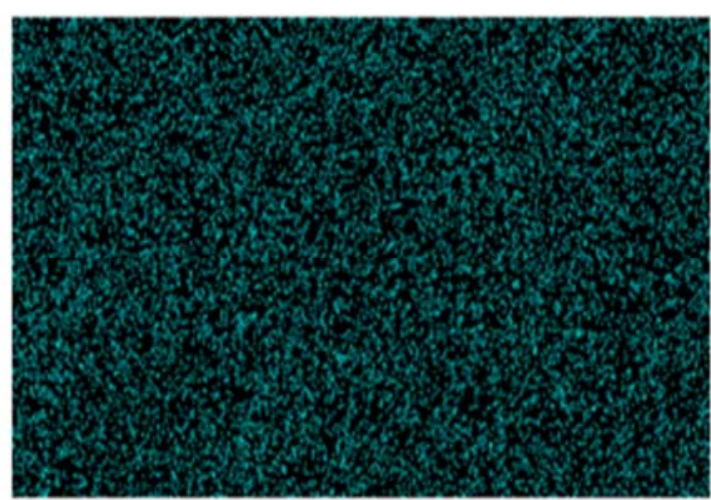

$\longdiv { 1 \mu \mathrm { m } }$

Cu La1_2

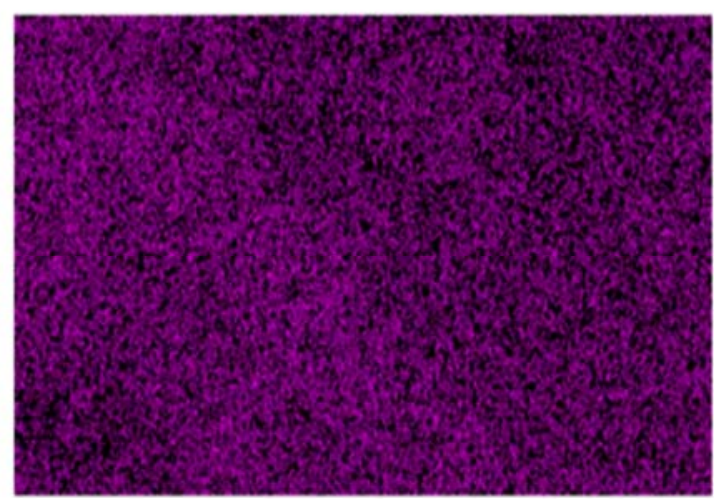

$\longdiv { 1 \mu \mathrm { m } }$ 
Zn L $\alpha 1 \_2$

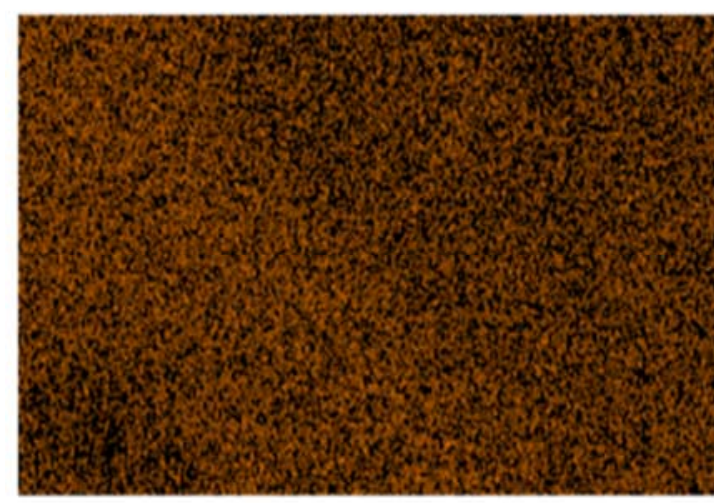

$\sqrt[1 \mu \mathrm{m}]{ }$
$\mathrm{O} K \alpha 1$

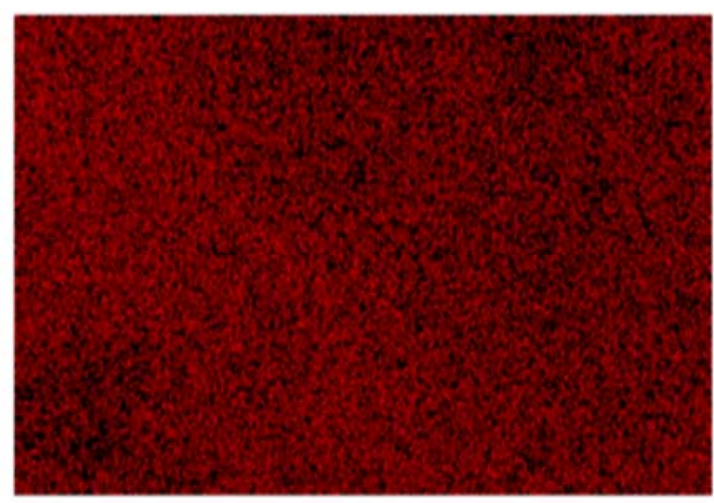

$\longdiv { 1 \mu \mathrm { m } }$

Figure S2. SEM image, EDXS layered image and EDXS elemental mapping in a chosen area $\# 2$ of undoped HEOx at a relatively high resolution (compared to Figure 1c).

2.3 EDXS elemental mapping in Li-doped HEOx

Electron Image 5

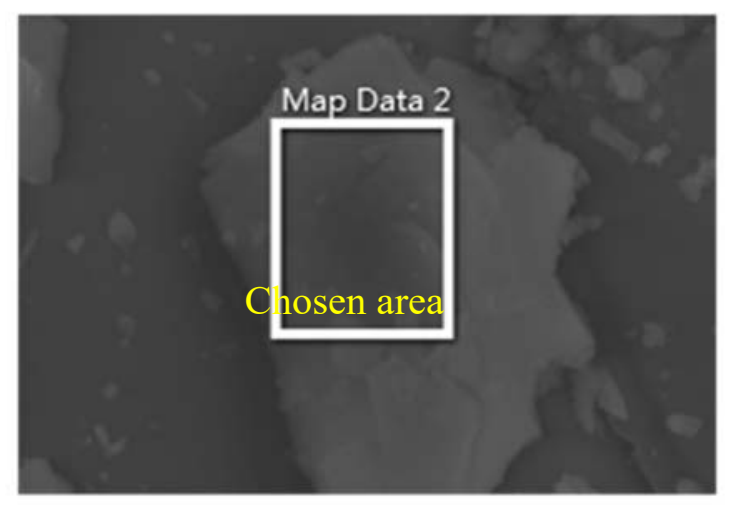

$2.5 \mu \mathrm{m}$
EDS Layered Image 2

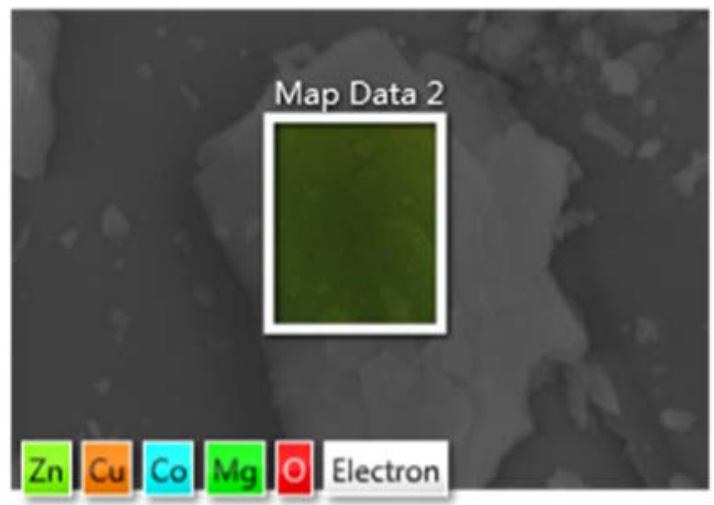

$2.5 \mu \mathrm{m}$ 


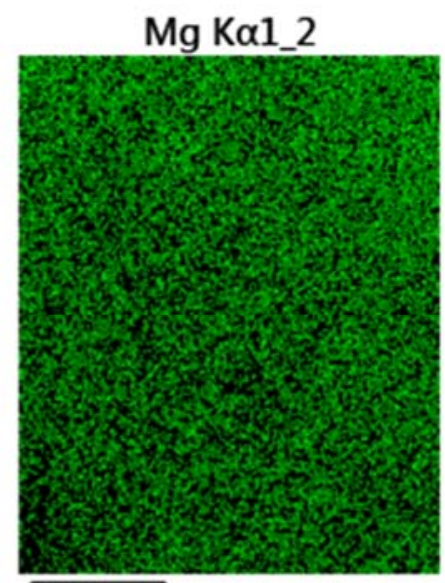

$\longdiv { 5 0 0 \mathrm { nm } }$

$\mathrm{Ni} K \alpha 1$

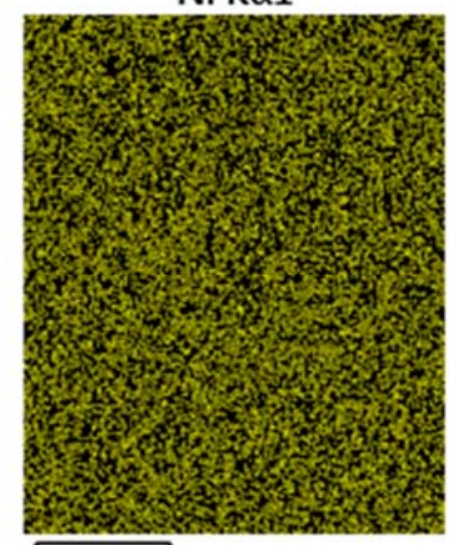

$\longdiv { 5 0 0 \mathrm { nm } }$

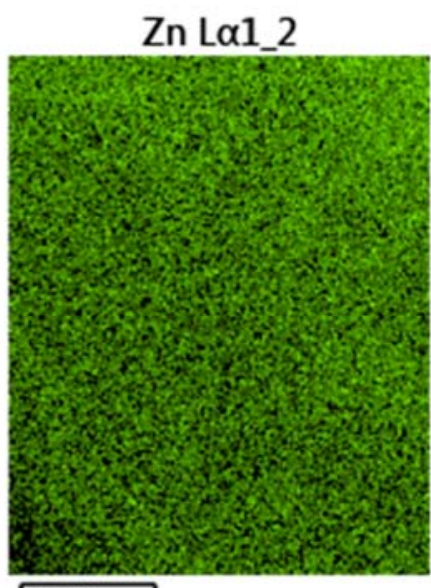

$500 \mathrm{~nm}$
Co $K \alpha 1$

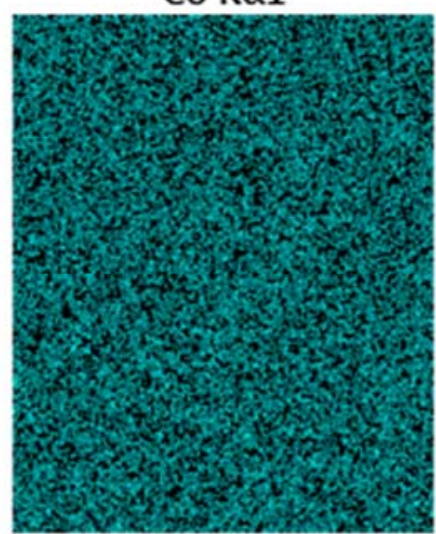

$\longdiv { 5 0 0 \mathrm { nm } }$

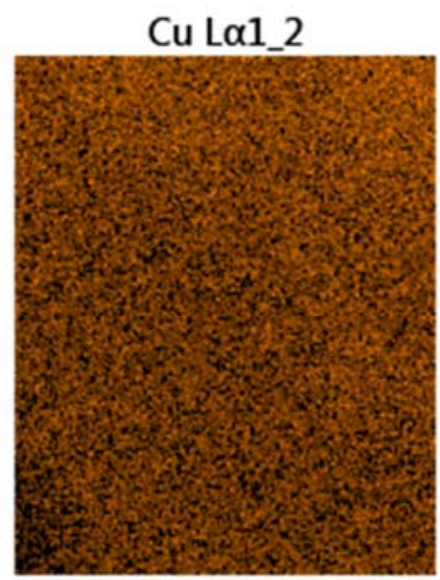

$\longdiv { 5 0 0 \mathrm { nm } }$

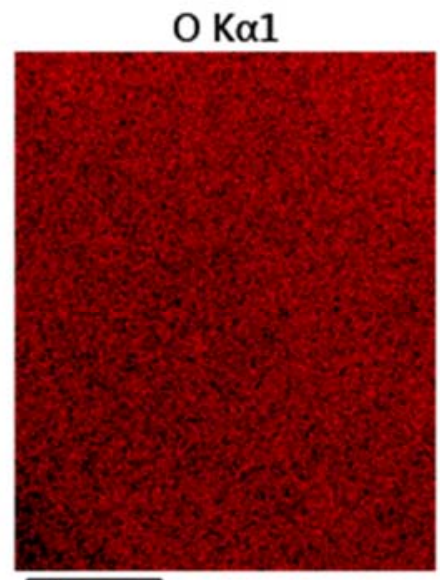

$\sqrt{500 \mathrm{~nm}}$

Figure S3. SEM image, EDXS layered image and EDXS elemental mapping in a chosen area of Li-doped HEOx at a relatively high resolution (compared to Figure $3 \mathrm{c}$ ). 
2.4 EDXS elemental mapping in Mn-doped HEOx

Electron Image 3

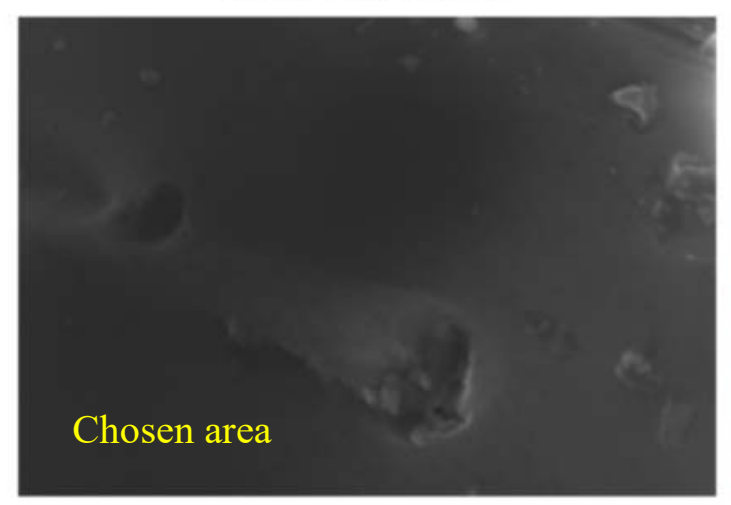

$\longdiv { 1 \mu \mathrm { m } }$

Mg Ka1_2

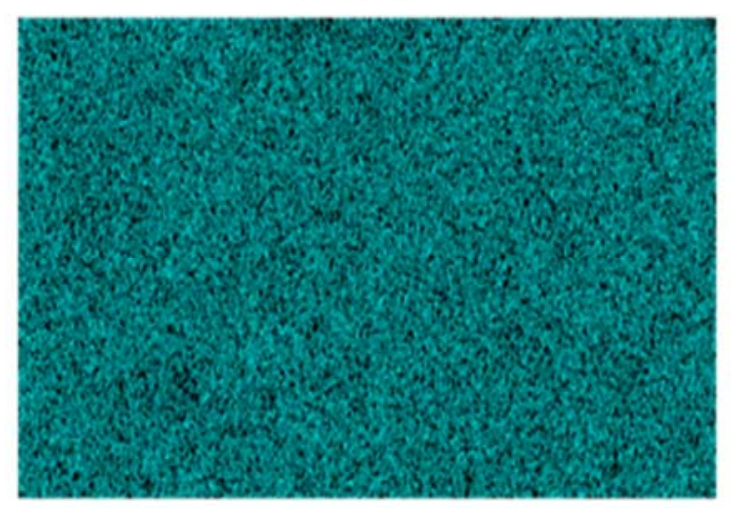

$2.5 \mu \mathrm{m}$
EDS Layered Image 1

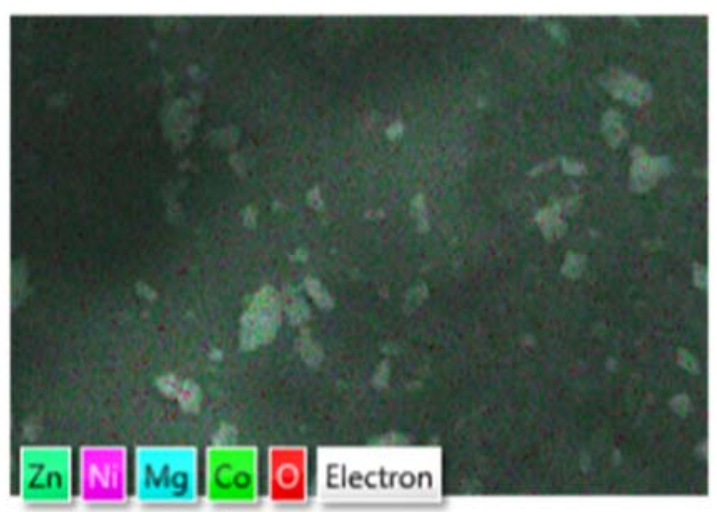

$\sqrt{2.5 \mu \mathrm{m}}$

Co $K \alpha 1$

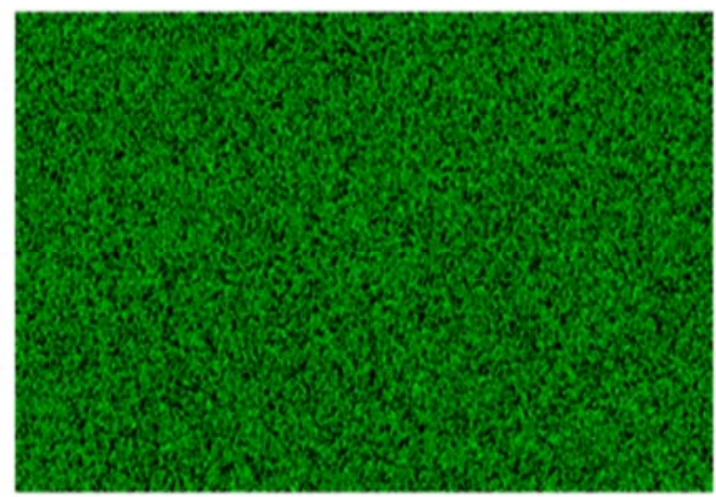

$2.5 \mu \mathrm{m}$ 
Ni K $\alpha 1$

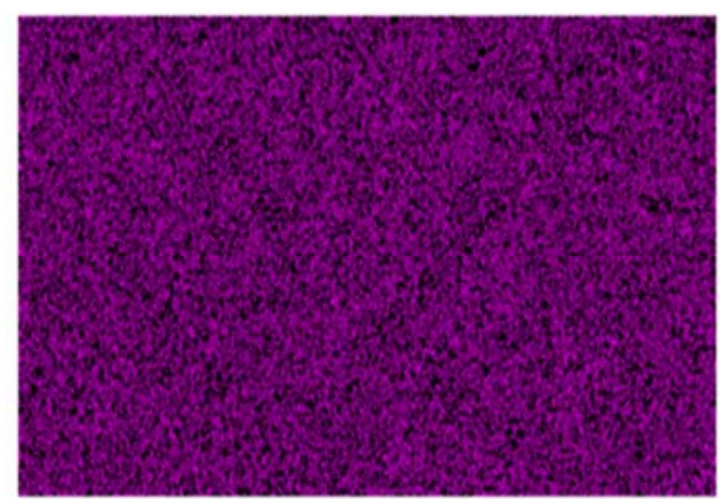

$2.5 \mu \mathrm{m}$

\section{Zn La1_2}

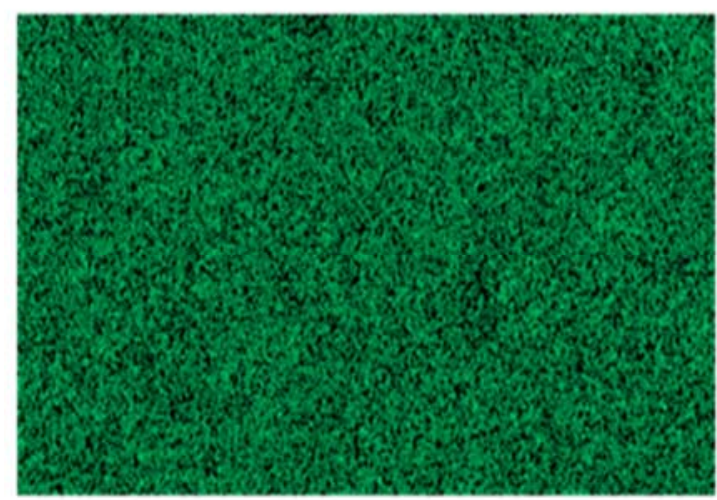

$2.5 \mu \mathrm{m}$
Cu La1_2

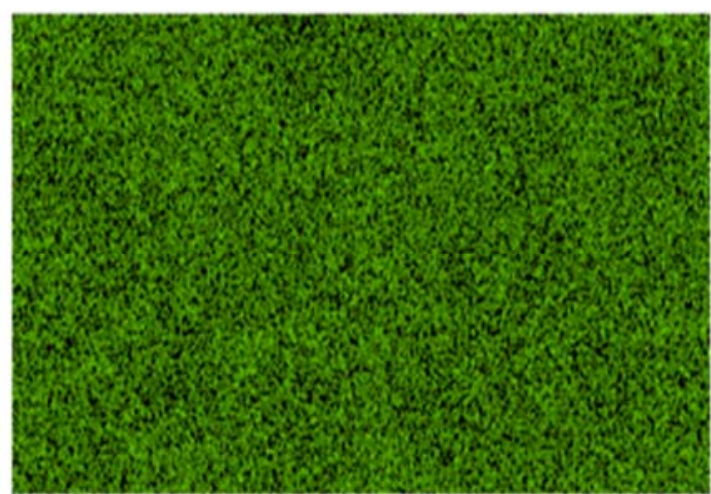

$2.5 \mu \mathrm{m}$

\section{Mn K $\alpha 1$}

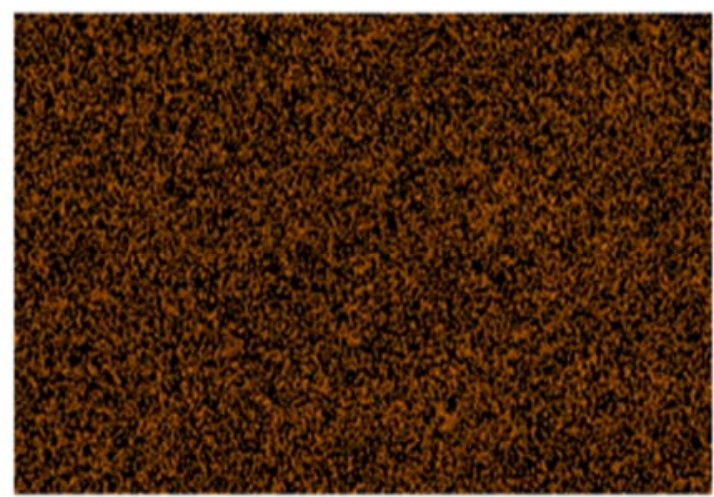

$2.5 \mu \mathrm{m}$

\section{$\mathrm{O} K \alpha 1$}

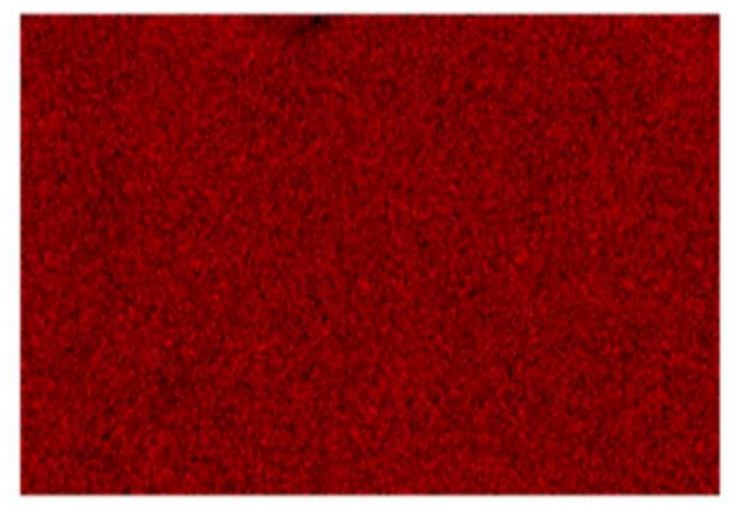

$2.5 \mu \mathrm{m}$

Figure S4. SEM image, EDXS layered image and EDXS elemental mapping in a chosen area of Mn-doped HEOx at a relatively high resolution (compared to Figure 4c). 
3. Additional high-pressure XRD patterns confirming mechanical stability of Li/Mn-doped high-entropy oxide

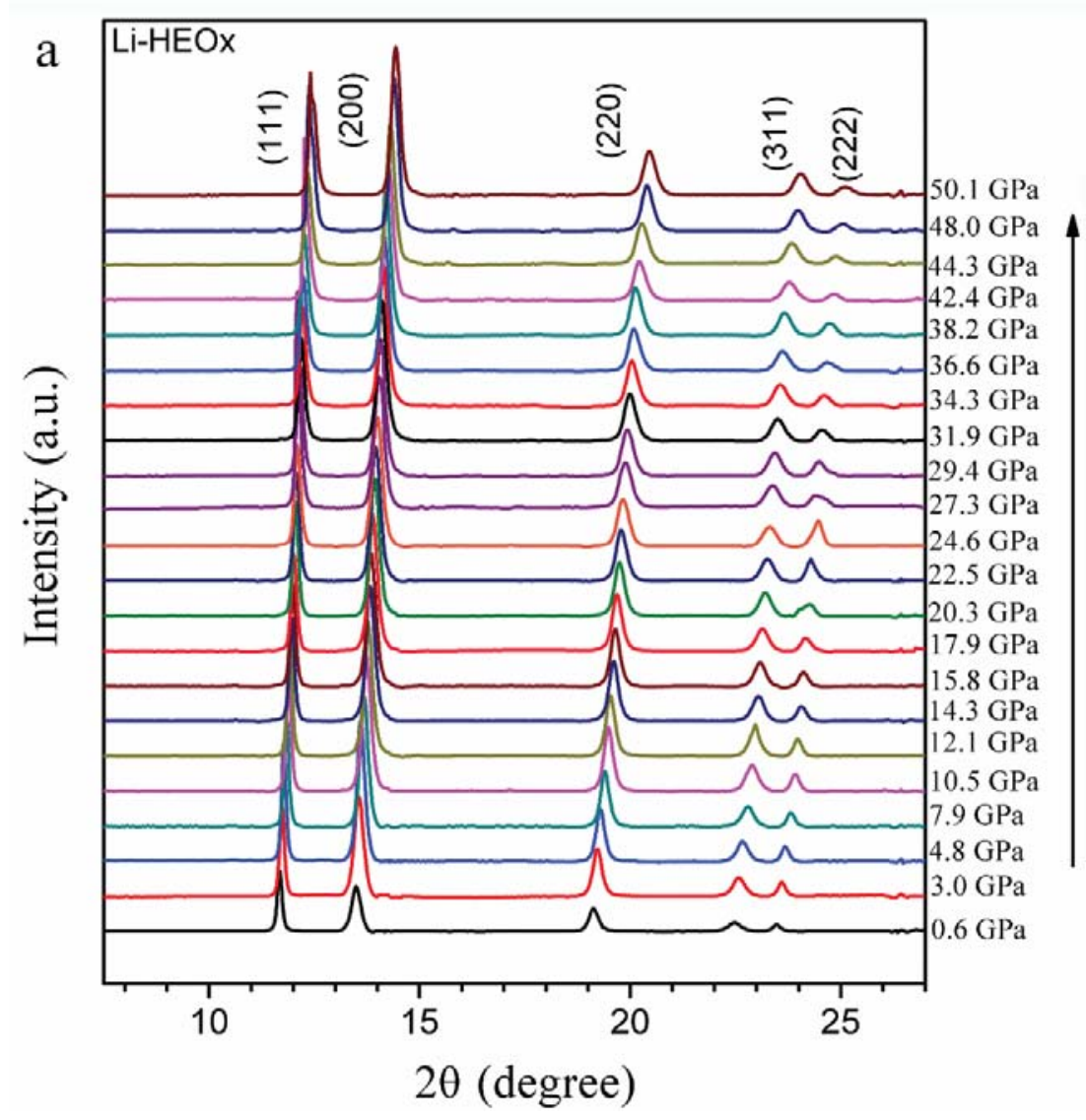




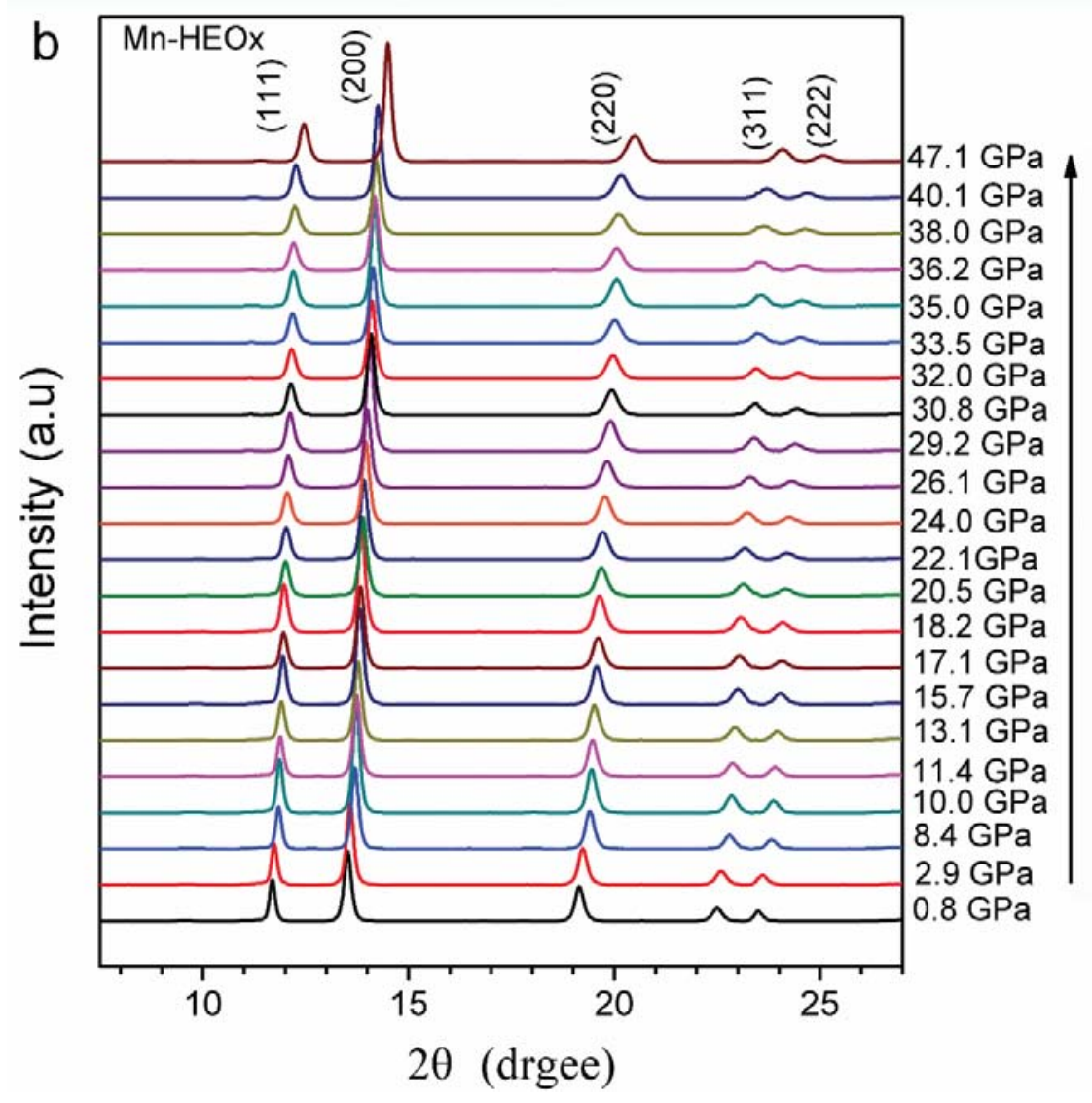

Figure S5. Additional high-pressure XRD patterns of Li-HEOx (a) and Mn-HEOx (b) from repeated synchrotron diffraction experiments. Pressure medium is methanol-ethanol mixture ( $4: 1$ in volume) and the X-ray wavelength is $0.4859 \AA$. 
4. Representative Rietveld fitting of XRD patterns of undoped and $\mathrm{Li} / \mathrm{Mn}$-doped high-entropy oxide
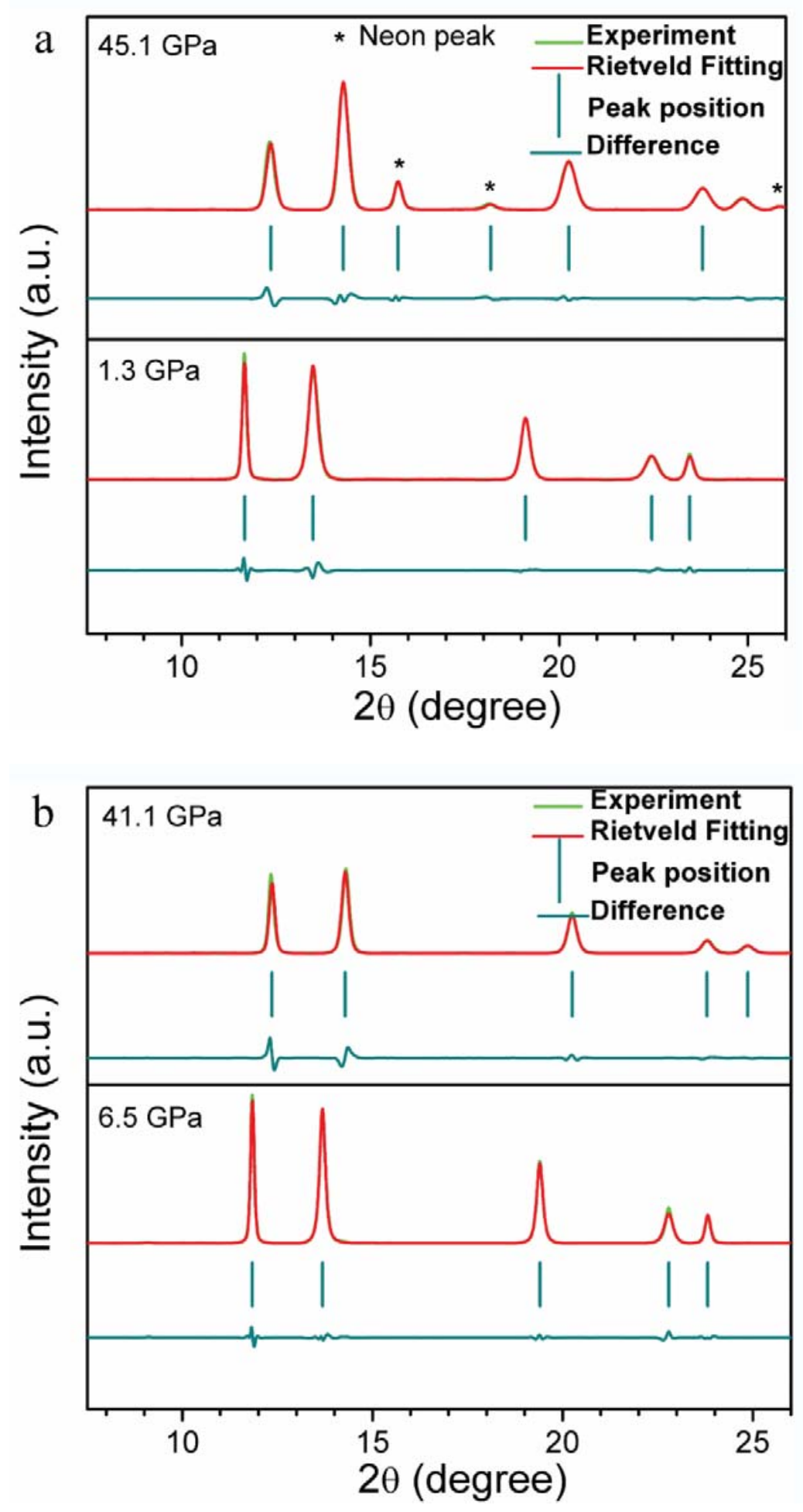


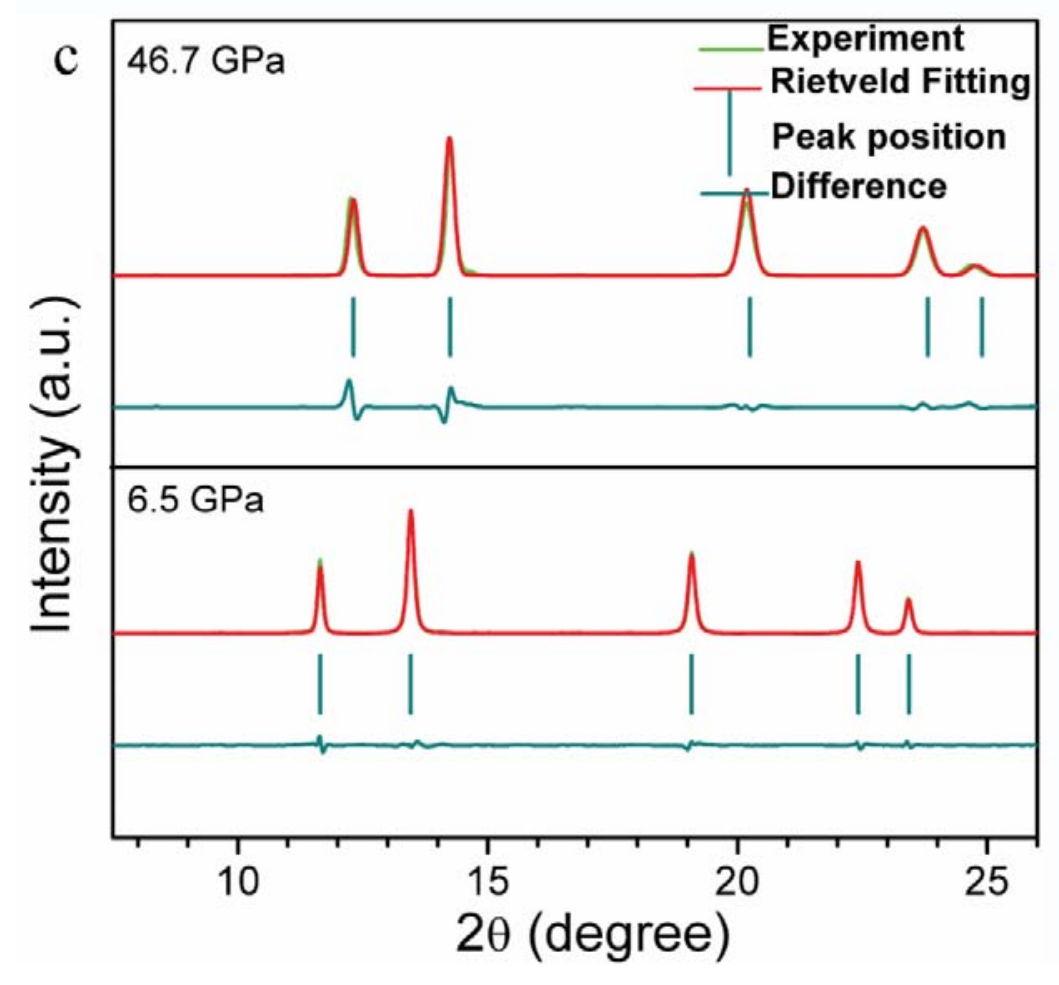

Figure S6. Representative Rietveld fitting of XRD patterns of u-HEOx (a), Li-HEOx (b) and Mn-HEOx (c) at different pressures using a structure model with a Fm $\overline{3} \mathrm{~m}$ space group.

\section{REFERENCES}

1. Feng, L. X.; Rong, C. X.; Fu, J. G.; Min, M. C. Ab Initio Calculations of Elastic Constants of $\mathrm{Li}_{2} \mathrm{O}$ under Pressure. Chin. Phys. Lett. 2006, 23, 925-927.

2. Pacalo, R. E.; Graham, E. K. Pressure and Temperature Dependence of the Elastic Properties of Synthetic MnO. Phys. Chem. Miner. 1991, 18, 69-80.

3. Liu, N. N.; Sun, J. L.; Wu, D. Elastic Constants and Thermodynamic Properties of Cu, $\mathrm{Cu}_{2} \mathrm{O}$ and $\mathrm{CuO}$ from First-Principles Calculations. Adv. Mater. Res. 2011, 335-336, 328-332.

4. Zha, C.-S.; Mao, H.; Hemley, R. J. Elasticity of $\mathrm{MgO}$ and a Primary Pressure Scale to 55 GPa. Proc. Natl. Acad. Sci. U. S. A. 2000, 97, 13494-13499.

5. Gale, J. D. GULP: Capabilities and Prospects. Z. Kristallogr. 2005, 220, 552-554.

6. Magnesium Oxide (MgO) Dielectric Constants, Optical and Photoelectric Properties. In II-VI and I-VII Compounds; Semimagnetic Compounds; Madelung, O., Rössler, U., 
Schulz, M., Eds.; Landolt-Börnstein - Group III Condensed Matter; Springer Berlin Heidelberg: Berlin, Heidelberg, 1999; pp 1-12.

7. Gražulis, S.; Daškevič, A.; Merkys, A.; Chateigner, D.; Lutterotti, L.; Quirós, M.; Serebryanaya, N. R.; Moeck, P.; Downs, R. T.; Le Bail, A. Crystallography Open Database (COD): An Open-Access Collection of Crystal Structures and Platform for World-Wide Collaboration. Nucleic Acids Res. 2012, 40, D420-D427. 